\title{
Characterization and localization of antigens for serodiagnosis of human paragonimiasis
}

\author{
Kurt C. Curtis ${ }^{1} \cdot$ Kerstin Fischer $^{1} \cdot$ Young-Jun Choi $^{2} \cdot$ Makedonka Mitreva $^{1,2} \cdot$ Gary J. Weil $^{1} \cdot$ Peter U. Fischer ${ }^{1}$ (D)
}

Received: 12 March 2020 / Accepted: 25 November 2020

(C) The Author(s) 2021

\begin{abstract}
Paragonimiasis is a foodborne trematode infection that affects 23 million people, mainly in Asia. Lung fluke infections lead frequently to chronic cough with fever and hemoptysis, and are often confused with lung cancer or tuberculosis. Paragonimiasis can be efficiently treated with praziquantel, but diagnosis is often delayed, and patients are frequently treated for other conditions. To improve diagnosis, we selected five Paragonimus kellicotti proteins based on transcriptional abundance, recognition by patient sera, and conservation among trematodes and expressed them as His-fusion proteins in Escherichia coli. Sequences for these proteins have 76-99\% identity with amino acid sequences for orthologs in the genomes of Paragonimus westermani, Paragonimus heterotremus, and Paragonimus miyazakii. Immunohistology studies showed that antibodies raised to four recombinant proteins bound to the tegument of adult $P$. kellicotti worms, at the parasite host interface. Only a known egg antigen was absent from the tegument but present in developing and mature eggs. We evaluated the diagnostic potential of these antigens by Western blot with sera from patients with paragonimiasis (from MO and the Philippines), fascioliasis, and schistosomiasis, and with sera from healthy North American controls. Two recombinant proteins (a cysteine protease and a myoglobin) showed the highest sensitivity and specificity as diagnostic antigens, and they detected antibodies in sera from paragonimiasis patients with early or mature infections. In contrast, antibodies to egg yolk ferritin appeared to be specific marker for patients with adult fluke infections that produce eggs. Our study has identified and localized antigens that are promising for serodiagnosis of human paragonimiasis.
\end{abstract}

Keywords Immunodiagnosis $\cdot$ Paragonimiasis $\cdot$ Paragonimus $\cdot$ Trematode $\cdot$ Recombinant antigens

\section{Introduction}

Foodborne trematode (FBT) infections are important neglected tropical diseases (NTDs) that affect about 56 million people and cause significant morbidity (Furst et al. 2012). Among FBT infections, lung flukes of the genus Paragonimus are arguably the most important group with an estimated 23 million human infections (Keiser and Utzinger 2009). Paragonimus

Section Editor: Sabine Specht

Peter U. Fischer

Pufischer@wustl.edu

1 Division of Infectious Diseases, Department of Medicine, Washington University School of Medicine, 4444 Forest Park Blvd, St. Louis, MO 63110, USA

2 McDonnell Genome Institute, Washington University School of Medicine, St. Louis, MO 63110, USA species are widely distributed in animals in Asia, Africa, and the Americas, but the infection risk for humans depends largely on whether humans ingest raw or undercooked crustaceans that act as intermediate hosts. For Paragonimus westermani, ingestion of raw meat from mammalian paratenic hosts provides an additional route of infection (Blair 2014). Human Paragonimus infection can be efficiently treated with a short course of praziquantel, but diagnosis is challenging, because infected people are often misdiagnosed and treated for pneumonia, tuberculosis, or cancer (Fischer and Weil 2015).

Paragonimus kellicotti is the only Paragonimus species endemic in the USA. Human P. kellicotti infections are rare, but in recent years, a number of serious cases have been reported from MO and adjacent states (Bahr et al. 2017; Lane et al. 2012). While the symptoms usually start 2 to 16 weeks after crayfish ingestion, accurate diagnosis is often delayed for a period of weeks to many months after the onset of symptoms (Lane et al. 2012). In order to improve the serological diagnosis of $P$. kellicotti infection, we developed a small animal 
Table 1 Summary of the five $P$. kellicotti proteins expressed in E.coli. These proteins include cysteine protease-6 (CP-6), cystatin-2 (CYS-2), myoglobin-1 (MYO-1), a microbial defense protein (MDP), and an egg yolk ferritin (EYF). The molecular mass of the recombinant protein includes $4 \mathrm{kDa}$ of the His-tag

\begin{tabular}{lllll}
\hline Clone designation & $\begin{array}{l}\text { Transcript } \\
\text { (McNulty et al. 2014) }\end{array}$ & Top BLASTP & $\begin{array}{l}\text { Expected } \\
\text { size }(\mathrm{kDa})\end{array}$ & $\begin{array}{l}\text { GenBank } \\
\text { acc \# }\end{array}$ \\
\hline Cp-6 & Pk00527 & gi|67773374|gb|AAY81944.1| cysteine protease 6 (Paragonimus westermani) & 40.7 & AZZ10060 \\
Cys-2 & Pk140546 & gi|150404782|gb|ABR68549.1| cystatin-2 (Clonorchis sinensis) & 16.1 & AZZ10061 \\
Myo-1 & Pk120808 & gi|59895953|gb|AAX11352.1| myoglobin 1 (Paragonimus westermani) & 20.4 & AZZ10059 \\
Mdp & Pk131774 & gi|379991184|emb|CCA61804.1| MF6p protein, partial (Fasciola hepatica) & 12.4 & AZZ10062 \\
Eyf & Pk145008 & gi|13625997|gb|AAK35224.1|AF367368_1 yolk ferritin (Paragonimus & 23.6 & AZZ10063 \\
& & westermani) & &
\end{tabular}

infection model to provide adult worms for antigen production. The native adult worm antigen was used to develop a Western blot assay that was sensitive and specific for paragonimiasis (Fischer et al. 2011b, 2013). Unfortunately, the native parasite antigen is not widely available, and recombinant antigen-based antibody tests are preferable in terms of feasibility and reproducibility. Therefore, we used a systems biology approach that included transcriptome sequencing followed by assembly and annotation, immunoprecipitation using patients' sera, and proteomics to identify candidate antigens for serodiagnosis of paragonimiasis (McNulty et al. 2014). In this study, we build on our progress by further characterizing five of these antigens and evaluate their potential value for diagnosis of $P$. kellicotti and $P$. westermani infections. These included a cysteine protease ( $\mathrm{Pk} 00394$ txpt2; MK050848), a cystatin (Pk45107-txpt2; MK050849), a myoglobin (Pk34178-txpt1; MK050847), a microbial defense protein (Pk39524 txpt1; MK050850), and an egg yolk ferritin (Pk48313-txpt; MK050851) (McNulty et al. 2014). Four of these serodiagnostic antigen candidates were among the top
25 immunoreactive adult $P$. kellicotti proteins, show relatively little conservation in other helminth genera, and represent distinct protein families (McNulty et al. 2014). The egg yolk ferritin was a highly abundant protein in adult worm extract and was target of a previous immunoassay for $P$. westermani (Kim et al. 2002a, b). So far, only few Paragonimus proteins have been recombinantly expressed and evaluated for their serodiagnostic potential. Among these are, apart from the egg yolk ferritin, different cysteine proteases of P. westermani, Paragonimus pseudoheterotremus, and Paragonimus skrjabini (Kim et al. 2000; Park et al. 2001; Yang et al. 2004; Yoonuan et al. 2016; Yu et al. 2017). Paragonimus species contain an expanded number of cysteine protease family members, and many of them are secreted and highly immunogenic, making them promising diagnostic antigens (Blair et al. 2016).

We cloned and expressed the proteins in Escherichia coli, purified the recombinant proteins, and tested their serodiagnostic potential with sera from patients infected with $P$. kellicotti or $P$. westermani. As negative controls, we used
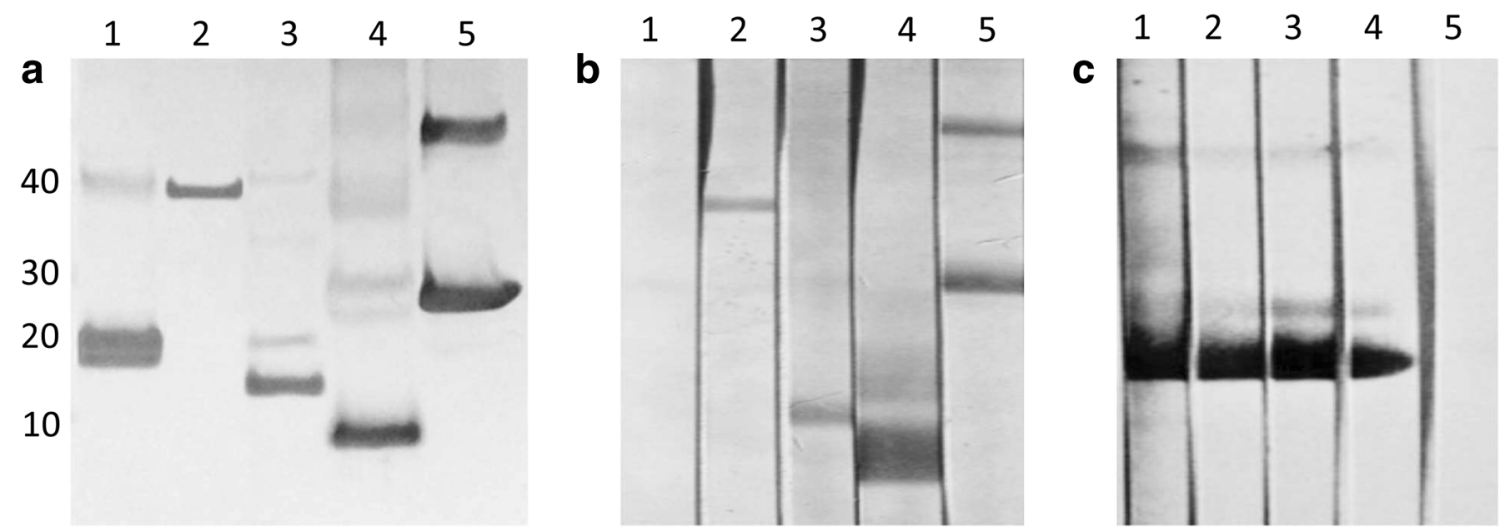

Fig. 1 Western blot analysis of recombinant (a, c) and native antigens (b). A recombinant antigen was run on a gel, blotted and probed using a monoclonal antibody against the His-tag on the recombinant antigen. The antibody detected the recombinant proteins at the expected size, but sometimes double bands were observed for example due to suboptimal reduction of protein and detection of dimers (lane 5). Lane 1, rMYO-1; lane 2, rCP-6; lane 3, rCYS-2; lane 4, rMDP; lane 5, rEYF. b $20 \mu \mathrm{g}$ of soluble whole worm extract was separated on a gel, blotted and probed

using polyclonal mouse antisera, generated against the recombinant protein. With exception of MYO-1, the antibodies recognized a native protein of the expected size. Lane 1, anti-rMYO-1; lane 2, anti-rCP-6; lane 3, anti-rCYS-2; lane 4, anti-rMDP; lane 5, anti-rEYF. c rMYO-1 antigen was run on a gel, blotted and probed using polyclonal mouse antiserum raised against rMYO-1 at different dilutions. Lane 1, 1000; lane 2, 1:2000; lane 3, 1:3000; lane 4, 1:4000; lane 5, negative control 


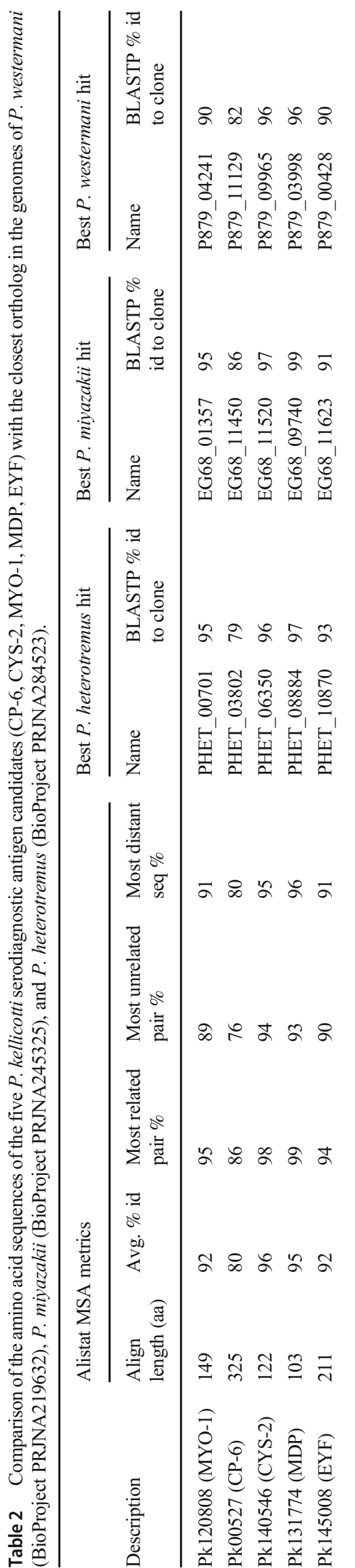

sera of patients infected with or without other trematode infections. In order to better characterize these target protein, we produced polyclonal mouse antibodies using recombinant protein and localized the antigens in adult $P$. kellicotti flukes.

\section{Material and Methods}

\section{Parasite material}

Adult stage $P$. kellicotti parasites were obtained as described previously from experimentally infected Mongolian gerbils, Meriones unguiculatus (Fischer et al. 2011b). The use of gerbils for $P$. kellicotti infections was approved by the Washington University Animal Studies Committee and followed the IACUC guidelines. Adult worms (45 days p.i.) were fixed in $4 \%$ buffered formalin for immunohistology studies or stored at $-80{ }^{\circ} \mathrm{C}$ for RNA extraction.

\section{Patient sera}

The study protocol was approved by the Human Research Protection Office at Washington University School of Medicine. This study included samples that were tested in our previous Western blot study with native $P$. kellicotti adult worm antigen (Fischer et al. 2013). Samples from patients with proven $P$. kellicotti infection were exclusively from MO, while samples with proven $P$. westermani infection (kindly provided by Dr. P. Wilkinson at the US Centers for Disease Control and Prevention) were from the Philippines.

\section{Protein expression and purification}

Total RNA was isolated from six adult $P$. kellicotti using the PureLink RNA mini kit (Thermo Fisher Scientific, Waltham MA, USA), and cDNA was synthesized using the 1 Script kit (BioRad, Hercules, CA, USA) according to the manufacturer's instructions. Diagnostic candidates were amplified with 50 ng of cDNA template and Platinum Pfx DNA polymerase (Thermo Fisher Scientific), and 20 pmol of primers (Supplemental Table S1). PCR products were Sanger sequenced and sequences of interest were submitted to GenBank

\section{Antigen sequence analysis}

The Sanger sequencing of the cDNA clones and the subsequent translation of the open reading frame generated protein sequences for comparative analysis (AZZ10059-AZZ10063). These sequences were used to identify orthologous sequences in other Paragonimus species (Rosa et al. 2020). The closest orthologs for P. kellicotti antigens in other Paragonimus species were retrieved by BLASTP against complete deduced 
proteomes that were generated by the Paragonimus genome sequencing project (Martin et al. 2015). Sequences are available at GenBank under BioProject id PRJNA284523 (P. heterotremus), PRJNA245325 (P. miyazakii), and PRJNA219632 ( $P$. westermani). In addition, we ran a blast search of the P. kellicotti $\mathrm{Cp}-6$ and Myo-1 against the orthologous groups from other FBTs and identified the top BLASTP hit. For Opisthorchis viverrini, no significant hit was obtained for the orthologous groups and we ran BLASTP against the GenBank NR database.

\section{Production and purification of recombinant $P$. kellicotti proteins}

PCR products were ligated into the directional pET100/DTOPO His-tagged expression vector (Invitrogen, Carlsbad, CA, USA) according to the manufacturer's instructions, and proteins were expressed using BL21 (DE3) E. coli cells (Sigma, St. Louis, MO, USA). Recombinant proteins were purified with His-Select Cobalt Affinity Get h8162 resin (Sigma).

\section{Western blot}

Western blot antibody assays were performed as previously described with $10 \mu \mathrm{g}$ of antigen protein per centimeter of nitrocellulose (NC) membrane. Test samples included sera from individuals infected with $P$. kellicotti, $P$. westermani, Schistosoma mansoni, or other helminths, and with sera from uninfected North Americans (Fischer et al. 2013). Individual $3 \mathrm{~mm}$ nitrocellulose (Invitrogen) strips were incubated with primary antibodies in mouse or human sera diluted 1:100 in PBS/Tween for $2 \mathrm{~h}$. Strips were washed in PBS/Tween three times and incubated in alkaline phosphatase-conjugated anti-

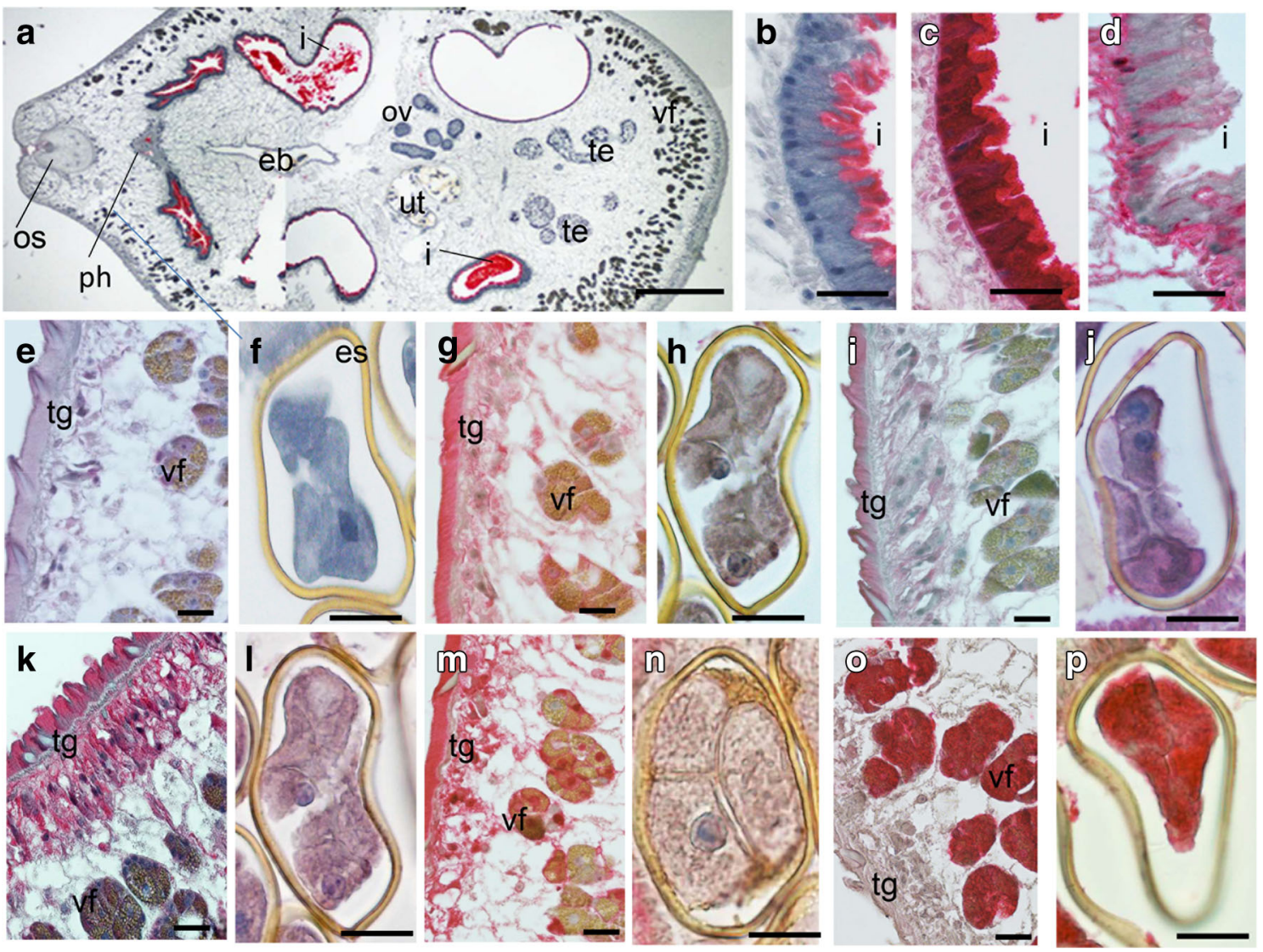

Fig. 2 Immunohistological localization of five candidate serodiagnostic antigens in adult $P$. kellicotti adult worms. Panels a, b, e, f Negative controls tested with a pre-immune mouse serum. No red staining was detected in any tissue except for the inner lining of the intestine $(\mathbf{a}, \mathbf{b})$. $\mathbf{c}, \mathbf{g}, \mathbf{h}$ Localization of CP-6 using a polyclonal mouse antibody shows intense staining of the intestinal wall (c) and the tegument, with weak staining of the vitelline glands (g). No staining was observed in the intrauterine eggs $(\mathbf{h}) . \mathbf{i}, \mathbf{j}$ Localization of CYS-2 shows moderate to weak staining of the tegument and vitelline glands (i) and the eggs (j).

$\mathbf{k}, \mathbf{l}$ Localization of MYO-1 shows strong staining of the tegument and weaker labeling of the vitelline gland (k) and the eggs (I). d, m, n Localization of MDP shows strong staining of the inner part of the intestinal epithelium (d), the tegument and the vitelline glands (m) but little staining in the eggs (n). o, p Localization of EYF shows strong labeling limited to the vitelline glands (o) and the eggs (p). Os, oral sucker; ph, pharynx; eb, excretory bladder; I, intestine; tg, tegument; ut, uterus; te, testis; ov, ovary; vf, vitelline follicle; es, egg shell. Scale bar for a is $500 \mu \mathrm{m}$ and for all others $20 \mu \mathrm{m}$ 
IgG or anti-IgG4 secondary antibodies (SouthernBiotech, Birmingham, AL, USA) diluted in PBS/Tween for $1 \mathrm{~h}$ at room temperature. After washing, antibody binding was revealed by incubating the NC strips in NBT/ BCIP (Fischer et al. 2013).

\section{Production of antibodies to recombinant proteins}

Polyclonal antibodies were raised in BALB/c mice by s.c. immunization with $20 \mu \mathrm{g}$ of recombinant proteins (rCP-6, rMYO-1, rCYS-2, rMDP, and rEYF) in complete Freund's adjuvant (1st immunization) or in incomplete Freund's adjuvant (boosting injections). Sera were stored at $-20^{\circ} \mathrm{C}$ until use. These antibodies served as positive controls for Western blot assays to verify that target antigens were present on $\mathrm{NC}$ membranes.

\section{Immunolocalization of recombinant antigens}

Formalin-fixed, adult $P$. kellicotti flukes were embedded in paraffin and sectioned at $5 \mu \mathrm{m}$. Sections were stained with hematoxylin and eosin to delineate general morphology. Two blocks with three flukes per block were sectioned, stained, and analyzed. The alkaline phosphatase-anti-alkaline phosphatase (APAAP) technique was used as previously described for immunostaining (Fischer et al. 2011a, 2017). Briefly, the unstained tissue sections were rehydrated and incubated in 10\% bovine serum albumin (Sigma) for $30 \mathrm{~min}$ to reduce background staining. Sections were then incubated with murine antibodies to $P$. kellicotti proteins at dilutions of 1:50 to $1: 200$ in phosphate-buffered saline containing $0.1 \%$ Triton- $\mathrm{X}$ and $0.1 \%$ bovine serum albumin. A dilution of 1:100 of the primary antibodies resulted in the best signal to noise ratio and was used for all further immunostainings. Polyclonal rabbit anti-mouse IgG (Dako, Carpinteria CA, USA) was applied as the secondary antibody, followed by incubation with APAAP (1:40, Sigma). The chromogen Fast Red TR salt (Sigma) was used as the substrate, and hematoxylin (Merck, Darmstadt, Germany) served as the counter-stain. The slides were examined with an Olympus-BX40 microscope (Olympus, Tokyo, Japan) and photographed with an Olympus DP70 digital camera.

For fluorescence microscopy, Alexa Fluor 488 anti-mouse IgG (Invitrogen, green fluorescence) was used as the secondary antibody, and wheat germ agglutinin $633(200 \mu \mathrm{g} / \mathrm{ml}$, Invitrogen, red fluorescence), and DAPI (Prolong Antifade with DAPI, Molecular Probes by Life Technologies, Carlsbad, CA, USA, blue fluorescence) were used to label membranes and double-stranded DNA, respectively (Fischer et al. 2017; McNulty et al. 2017). Sections were examined with a wide field fluorescence microscope (WFFM, Zeiss Axios Imager Upright Fluorescence Microscope) with planapochromat $\times 100$ oil, $\times 63$, or $\times 40$ objectives.

\section{Results}

\section{Expression of immunoreactive proteins}

cDNAs of five $P$. kellicotti immunoreactive antigens were chosen for expression: a cysteine proteinase (CP-6), a myglobin (MYO-1), a cystatin (CYS-2), a microbial defense protein (MDP), and an egg yolk ferritin (EYF) (Table 1). The recombinant proteins had the expected molecular masses (Fig. 1). However, rMYO-1 showed a more diffuse band, which could be caused by the presence of a second, slightly smaller
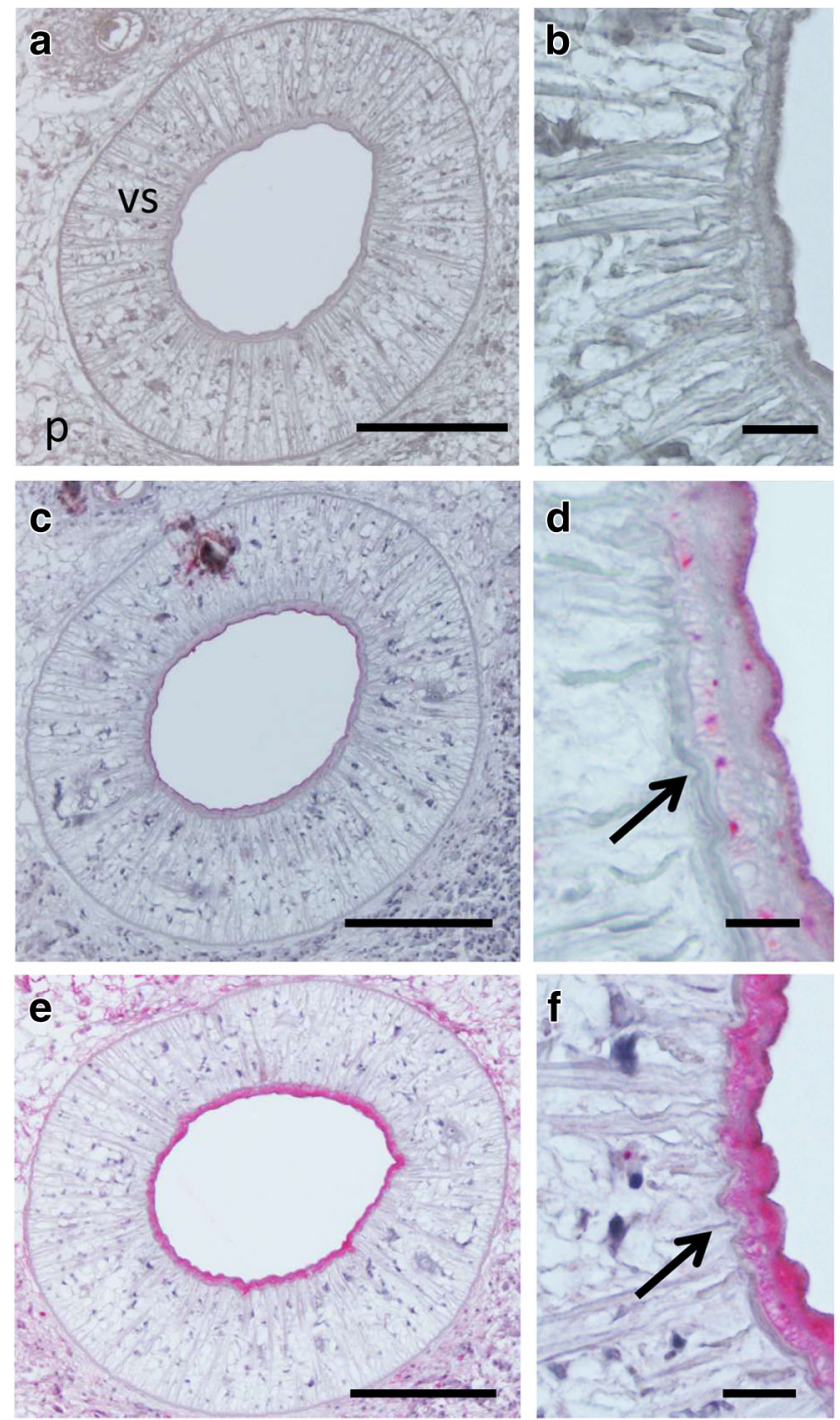

Fig. 3 Immunohistological localization of antigens with serodiagnostic potential in the ventral sucker of adult $P$. kellicotti flukes using the APAAP method. a, b Negative control using a pre-immune serum of a mouse used for antibody generation shows no staining. $\mathbf{c}, \mathbf{d}$ Localization of CP-6 shows staining of the outer part of the ventral sucker and some granular staining of single cells (arrow). No staining was observed in the parenchyma. e, f Localization of MDP shows intense staining of the entire inner part of the ventral sucker and weaker staining in the parenchyma. Vs, ventral sucker; p, parenchyma. Scale bar for A, C, E is $100 \mu \mathrm{m}$ and for B, D, E $10 \mu \mathrm{m}$ 
protein, or partial degradation. The antibody directed against the His-tag recognized rEYF at approximately $24 \mathrm{kDa}$ and a second band at approximately $48 \mathrm{kDa}$ indicative of incomplete reduction and detection of a dimer. When we tested polyclonal antibodies raised against the recombinant proteins by Western blot with crude adult worm extract as antigen, we obtained similar results for CP-6, CYS-2, MDP, and EYF with lower $\mathrm{kDa}$ values (consistent with the absence of the His-tag in the native proteins). However, antibodies raised to rMYO-1 did not react with antigens in the worm extract (Fig. 1b), but the antibodies bound to rMYO protein at the expected size (Fig. 1c).

\section{Conservation of selected immunoreactive proteins}

We compared the amino acid sequences of the five selected $P$. kellicotti proteins with orthologous proteins from the P. westermani, P.miyazakii, P. heterotremus genomes (Martin et al. 2018). Close orthologs of the P. kellicotti sequences for CP-6, MYO-1, CYS-2, MDP, and EYF were identified in the genomes of all three species with amino acid identities that ranged between 79 and 99\% (Table 2). These five proteins were all highly conserved between the four species, and there were only minor differences. MDP was the most highly conserved protein with identities between 96 and $99 \%$, while CP-6 was the least conserved protein with identities between 79 and $86 \%$. Still, this high degree of conservation of the selected serodiagnostic antigen candidates may facilitate the development of a pan-Paragonimus serodiagnostic antigen. This is supported by the fact that the selected antigen candidates are conserved not only among Asian, but also North American species.

\section{Immunolocalization of candidate diagnostic proteins in adult $P$. kellicotti}

All five antigens showed distinct localization patterns (Fig. 2). Pre-immune murine sera did not label tegument, ova (Fig. $2 \mathrm{e}, \mathrm{f})$, or other structures except from a part of the intestine. The inner lining of the intestine of $P$. kellicotti was labeled with all immune and negative control sera. This non-specific staining is probably because of endogenous alkaline phosphatase that is detected by the anti-alkaline phosphatase antibody component in the APAAP localization protocol (Fig. 2a, b). However, the distinct non-specific labeling of parts of the intestine was easily differentiated from specific antibody staining of the intestine (Fig. 2c, d). In agreement with this observation, no unspecific labeling was observed when Alexa Fluor 488 anti-mouse IgG was used as secondary antibody was used that does not contain an anti-alkaline phosphatase antibody like the APAAP complex.

CP-6 is mainly localized in the tegument at the parasitehost interface (Fig. 2g). Some staining was detected in the vitelline follicles, and strong staining was seen in the full thickness of the intestine (not limited to the inner lining) (Figs. 2c, 4d-f). Staining was also present in the oral and ventral suckers (Figs. 3c, d; 4a-c). CYS-2 was localized to the tegument, vitelline follicles, and intrauterine eggs (Fig.
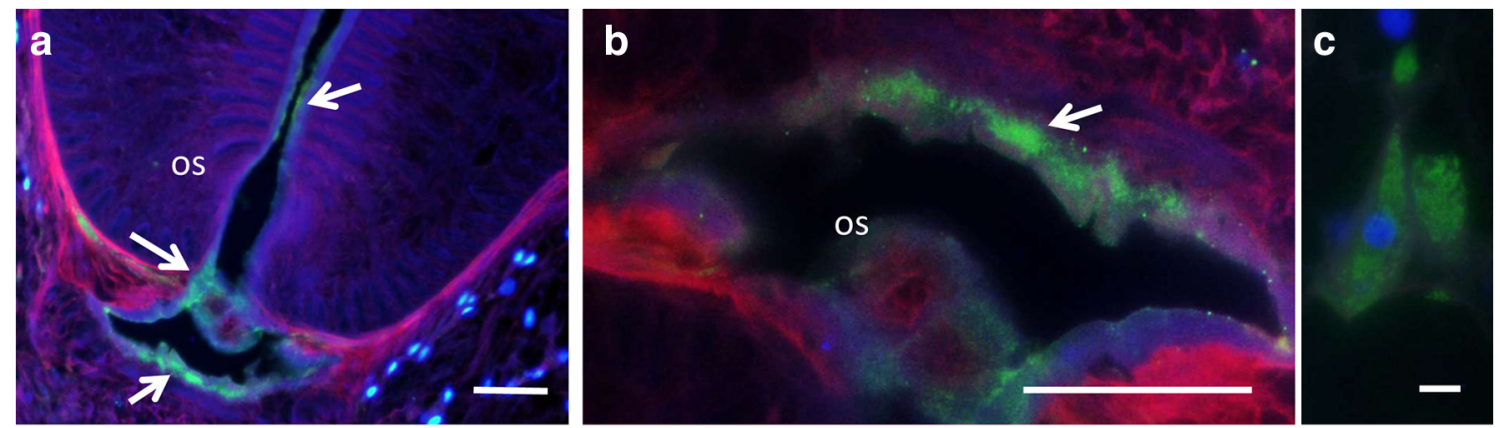
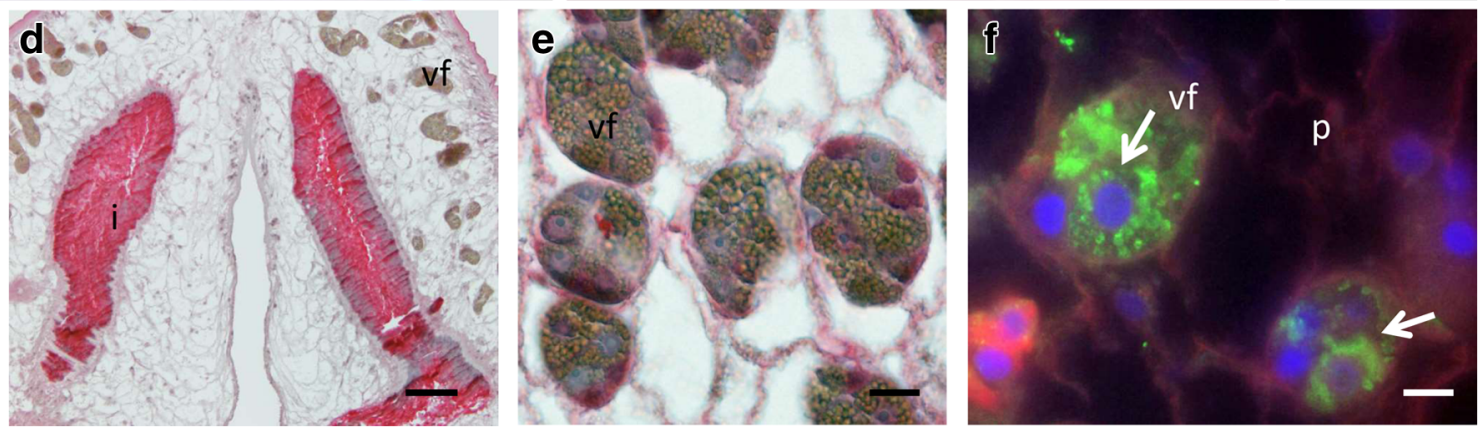

Fig. 4 More detailed immunohistological localization studies of CP-6 in adult $P$. kellicotti worms by immunofluorescence $(\mathbf{a}-\mathbf{c}, \mathbf{f})$ or APAAP $(\mathbf{d}$, e). a, b Intense green labeling of the mouth and the outer area of the oral sucker. c Distinct labeling of the cytosol of a cell close to the mouth. d
Intense staining of the intestine that exceeds the alkaline phosphatase background pattern in the intestine (compare Fig. 1 a, b). e, f Intense granular staining of the vitelline follicles. Os, oral sucker; vf, vitelline follicle; p, parenchyma. Scale bar for $\mathbf{a}, \mathbf{b}, \mathbf{d} 100 \mu \mathrm{m}$ and for $\mathbf{c}, \mathbf{f} 10 \mu \mathrm{m}$ 

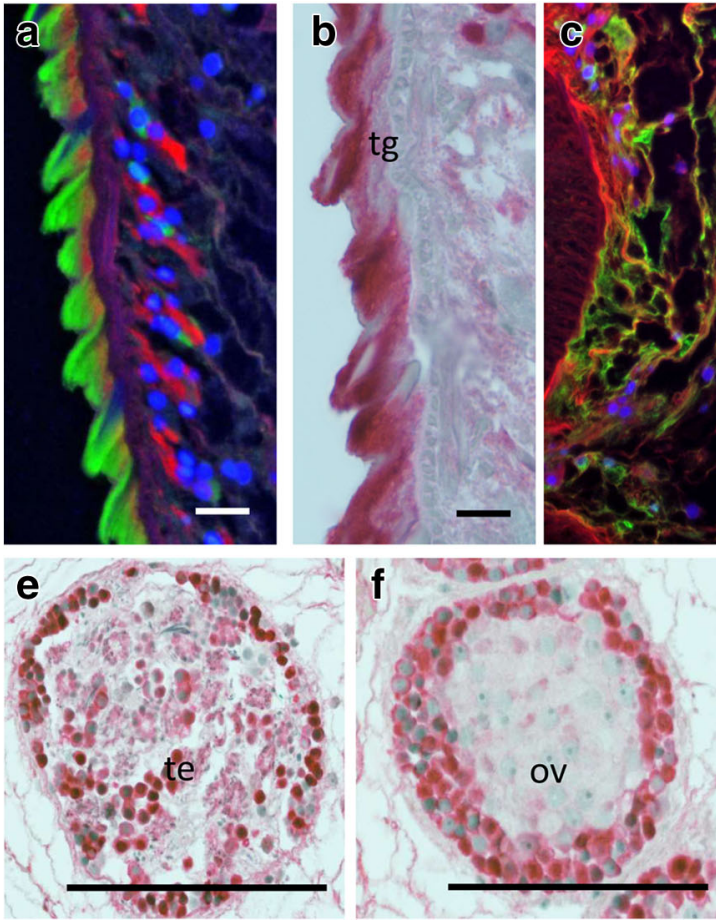

Fig. 5 More detailed immunohistological localization of MDP in adult $P$. kellicotti flukes using the immunofluorescence $(\mathbf{a}, \mathbf{c}, \mathbf{d}, \mathbf{h})$ or APAAP $(\mathbf{b}, \mathbf{e}-\mathbf{g})$. a, b Intense labeling of outer tegument. $\mathbf{c}$ Labeling of the parenchyma in vicinity of the oral sucker. d Intense staining of outer

$2 \mathrm{i}, \mathrm{j}$ ), while antibodies to MYO-1 bound strongly to the tegument. Weaker MYO-1 staining was observed in the parenchyma and in intrauterine eggs (Fig. 2k, 1).

Staining for MDP was widespread and it appears that this protein is abundant in many worm tissues (see Supplemental Figure 1). MDP staining was especially intense in the tegument, inner parts of the suckers, the intestine, and the parenchyma (Figs. 2d, m; 3e, f; 5). Staining was also present in the testis, ovaries, and the vitelline follicles (Fig. 4). Analysis of total adult $P$. kellicotti extract by Western blot using this antibody supports the finding that this is an abundant protein and that the antibody detects specifically only a single protein band at around $8.4 \mathrm{kDa}$.

Localization studies showed that EYF was abundant in the vitelline follicles and intrauterine eggs (Fig. 2o, p), but not in

Table 3 Results of the pilot evaluation of the serodiagnostic potential of five recombinant $P$. kellicotti proteins, cysteine protease-6 (CP-6), cystatin-2 (CYS-2), myoglobin-1 (MYO-1), a microbial defense protein
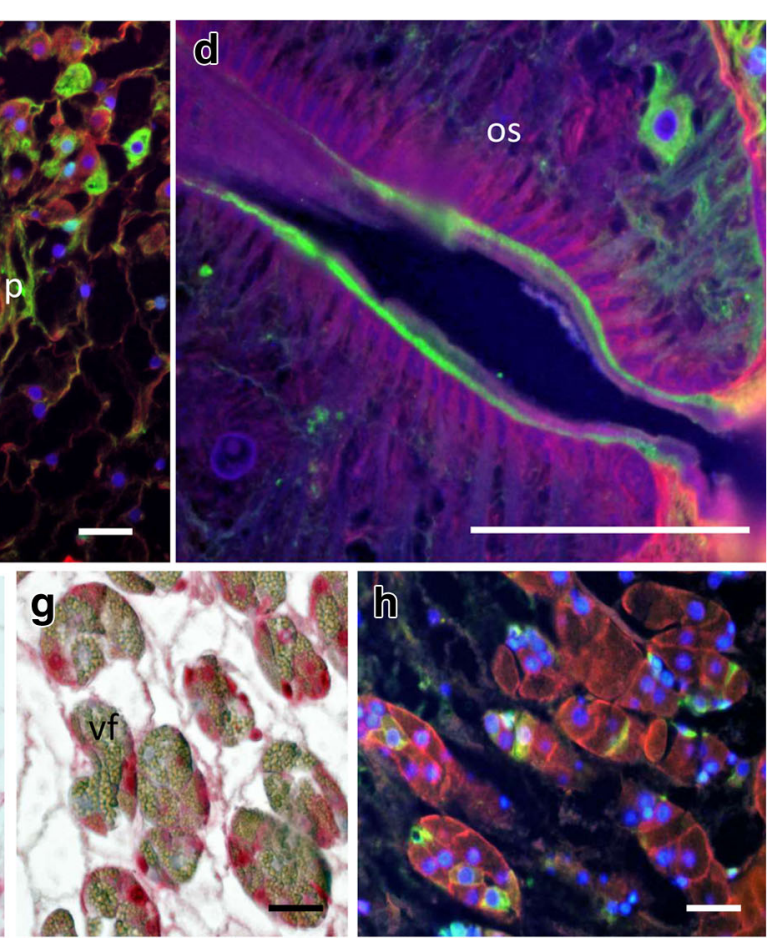

parts of the oral sucker. e, f Intense granular staining of testis and ovary. $\mathbf{g}, \mathbf{h}$ Granular staining of single cells within the vitelline follicle. Os, oral sucker; vf, vitelline follicle; $p$, parenchyma; tg, tegument; os, oral sucker; te, testis; ov, ovary. Scale bar for $\mathbf{a}, \mathbf{b}, \mathbf{c} 10 \mu \mathrm{m}$ and for $\mathbf{d}, \mathbf{e}, \mathbf{f} 10 \mu \mathrm{m}$

other tissues of $P$. kellicotti. These results show that immunolocalization is not only helpful for a better characterization of proteins of interest, but it also helps to experimentally identify proteins that are secreted and/or localized at the parasite-host interface, and maybe exposed to the human immune system and, therefore, may represent preferred targets for serodiagnostics.

\section{Evaluation of recombinant proteins as serodiagnostic antigens}

The diagnostic potential of the five antigen candidates was assessed by Western blot with a panel of sera from 17 subjects with proven Paragonimus infections (13 P. kellicotti cases from MO and $4 P$. westermani cases from the Philippines)

(MDP), and an egg yolk ferritin (EYF). For all proteins, total IgG antibodies were detected by Western blot, but for Cp- 6 antibodies of the IgG4, subclass was also assessed in order to increase specificity.

\begin{tabular}{|c|c|c|c|c|c|c|}
\hline \multirow[t]{2}{*}{ Sera type } & \multicolumn{2}{|l|}{$\mathrm{Cp}-6$} & \multirow{2}{*}{$\begin{array}{l}\text { Cys-2 } \\
\text { IgG, pos/N }\end{array}$} & \multirow{2}{*}{$\begin{array}{l}\text { Myo-1 } \\
\text { IgG, pos/N }\end{array}$} & \multirow{2}{*}{$\begin{array}{l}\text { Mdp } \\
\text { IgG, pos/N }\end{array}$} & \multirow{2}{*}{$\begin{array}{l}\text { Eyf } \\
\text { IgG, pos/N }\end{array}$} \\
\hline & $\operatorname{IgG}, \operatorname{pos} / \mathrm{N}$ & $\mathrm{IgG} 4, \operatorname{pos} / \mathrm{N}$ & & & & \\
\hline P. kellicotti & $13 / 13$ & $13 / 13$ & $4 / 13$ & $13 / 13$ & $10 / 13$ & $6 / 13$ \\
\hline P. westermani & $4 / 4$ & $4 / 4$ & $0 / 4$ & $4 / 4$ & $3 / 4$ & $1 / 4$ \\
\hline Other helminth infections & $5 / 5$ & $0 / 23$ & $0 / 5$ & $0 / 23$ & $0 / 5$ & $0 / 5$ \\
\hline US non-infected & $1 / 1$ & $0 / 5$ & $0 / 2$ & $0 / 5$ & $0 / 2$ & $0 / 2$ \\
\hline
\end{tabular}


and with sera from Paragonimus-negative controls with or without other trematode infections (Table 3 ). In this pilot evaluation, CP-6 and MYO-1 had a sensitivity of $100 \%$ with sera from paragonimiasis patients vs sensitivities of 76,41 , and $24 \%$ for MDP, EYF, and CYS-2, respectively. All clinical cases had antibodies reactive to antigens present in total adult worm extract. Some of the infected subjects had detectable eggs in sputum or stool. In total, 7 P. kellicotti and 4 $P$. westermani infected subjects had detectable eggs, and of those, 6 P. kellicotti and $1 P$. westermani samples had antibodies reactive with recombinant EYF by Western blot. None of the 28 control sera from persons with no infection or with infections with other helminth parasites had antibodies to Myo-1. MDP, EYF, and CYS-2 also appeared to have good specificity, but fewer negative control sera were tested with these antigens because of their sub-optimal sensitivity.

All sera from paragonimiasis patients contained $\operatorname{IgG}$ antibodies to CP-6, but six control sera also had antibodies that reactivated with the antigen (Fig. 6a). Specificity improved to $100 \%$ with no loss of sensitivity when we used anti-human IgG4 as the secondary antibody in CP-6 (Table 3; Fig. 6b).

We performed CP-6 IgG4 Western blots with paired serum samples from four patients with $P$. kellicotti infections that were collected before and after they were successfully treated with praziquantel. Treatment success was defined as resolution of clinical symptoms associated with infection. One patient's sera had similar band intensities before and 53 days post-treatment. However, band intensities decreased 224, 42, and 59 days after treatment in three other patients (Fig. 6c). These results suggest that although IgG4 antibody titers to CP6 tend to decrease after successful treatment, they sometimes remain detectable for some months.

\section{Discussion}

Human paragonimiasis in an important FBT infection, and it was estimated that in 2017, this group of NTDs is responsible for 1,870,700 years lived with disabilities (YLD) (GBD 2017 Disease and Injury Incidence and Prevalence Collaborators 2018). Among the FBT infections, about $50 \%$ of the YLD are assumed to be caused by paragonimiasis (Furst et al. 2012). However, these estimates do not include infections in Africa or North America, and because of underdiagnosis and underreporting, the global number of infections is likely to be higher. Furthermore, pathogenicity and the ascribed disability weight for paragonimiasis is species-dependent and may also be an underestimate (Feng et al. 2018). Therefore, improved diagnosis for paragonimiasis would be important not only for clinical care, but also for a more accurate estimation of its public health importance.

We amplified, expressed, characterized, and evaluated five recombinant $P$. kellicotti proteins for their potential as serodiagnostic antigens. Two proteins, CYP-6 and MYO-1, show great potential as serodiagnostic antigens for paragonimiasis. Immunolocalization studies showed that four of the five candidate diagnostic antigens were present in the tegument of adult worms, at the host-parasite interface. Protein EYF was only detected in the vitellaria and the eggs, and it is possible that antibodies to this protein only develop after worms have matured to adulthood with egg production.

Few proteins have been localized in adult Paragonimus flukes before, and our thorough immunolocalization of five proteins with antibodies raised against recombinant proteins has provided significant new information. MYO-1 was previously localized in $P$. westermani, and our data confirm those
Fig 6 Western blot detection of antibodies to $\mathrm{rCP}-6$ in patient and control sera. a Detection of total IgG antibodies. b Detection of $\mathrm{IgG}_{4}$ subclass antibodies. Lanes 1-10 and 12-14 sera are from individuals with $P$. kellicotti infection; lanes 11, 15-17 sera are from individuals with $P$. westermani infection; lanes 18-23, tested sera were from individuals with Fasciola hepatica or Schistosoma mansoni infections. c Western blot analysis of selected patient sera before and after successful treatment using rCP-6 as antigen. Lanes 1, 3, 5, and 7 before treatment and lanes 2, 4, 6, 8, 53 days, 224 days, 42 days, and 59 days after treatment

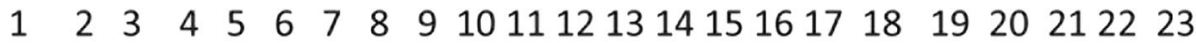

a

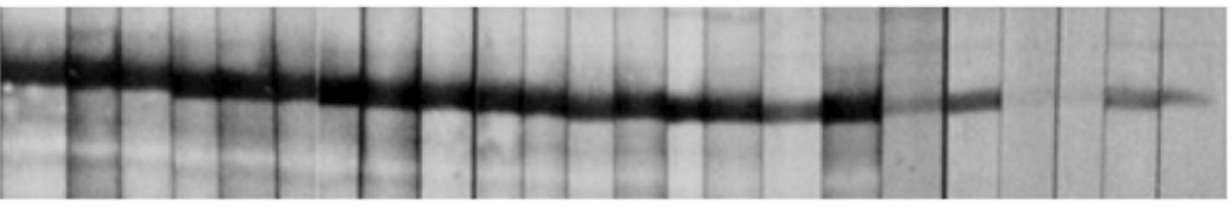

b
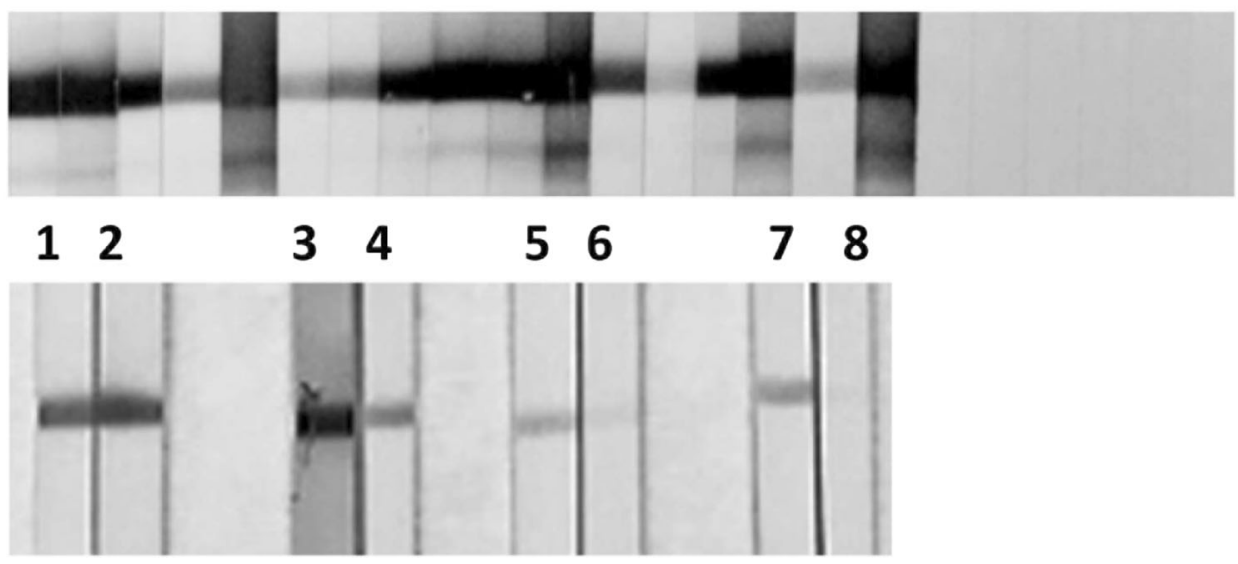
findings with strong staining throughout the parenchymal tissue and the tegument, and absent or weak staining of the embryonic cells inside the eggs (de Guzman et al. 2007). There are many different cysteine proteases in Paragonimus, and their tissue localization appears to vary. Prior studies have localized them in the intestinal epithelium (Choi et al. 2006), esophagus (Yoonuan et al. 2016), and the tegument in the anterior part of the fluke including the oral sucker (Na et al. 2006).

Based on recently generated genomic information, all five of the proteins selected for this study showed a high degree of conservation between four Paragonimus species (three from Asia and one from North America). Although MYO-1 of P. kellicotti is $90-95 \%$ identical to the orthologs in the genomes of the other three Paragonimus species, it is only 53\% and 58\% identical to orthologs in the genomes of the related trematodes Clonorchis sinensis and O. viverrini, respectively (Supplemental Table S2) (Wang et al. 2011; Young et al. 2014). Similarly, CP-6 of P. kellicotti is $79-86 \%$ identical to orthologs in the genomes of the other three Paragonimus species, but only $49 \%$ and $55 \%$ identical to the closest orthologs in the genomes of the related trematodes $C$. sinensis and $O$. viverrini, respectively (Supplemental Table S2). Even lower degrees of similarities were observed for other trematode species such as Fasciola gigantica, Fasciola hepatica, and Schistosoma mansoni. Therefore, the relatively high genusspecific conservation in Paragonimus may facilitate the development of pan-Paragonimus serology tests.

Few Paragonimus proteins have been evaluated as potential serodiagnostic antigens, and most have been evaluated with sera from persons infected with a single Paragonimus species. The EYF antigen of $P$. westermani was reported to have high specificity (Kim et al. 2002b). However, in experimentally infected cats, it took 13 weeks following infection before antibodies were detectable by Western blot. Our study confirmed the high specificity of this antigen, but showed unsatisfactory sensitivity, especially for sera from persons with early infections. Myoglobins are highly abundant proteins in adult P. westermani and P. kellicotti (de Guzman et al. 2007; McNulty et al. 2014), but they have not been previously studied as diagnostic antigens for paragonimiasis. While cystatins have not been tested for their direct serodiagnostic potential, a cystatin capture assay has been used to capture cysteine proteinases as diagnostic antigens (Ikeda 1998).

Cysteine proteases have been suggested as targets for serodiagnosis of paragonimiasis (Blair et al. 2016). Cysteine protease fractions have been partially isolated from excretory/ secretory products from $P$. westermani, and this antigen preparation showed increased specificity compared to a total worm extract (Ikeda et al. 1996). Paragonimus species have many cysteine proteases, and only a few of them have been examined as diagnostic antigens. For example, eleven cathepsin $\mathrm{F}$ family cysteine proteinases of $P$. westermani were reported to have diagnostic sensitivities that ranged between 38.4 and $84.5 \%$ with specificities above $87 \%$ (Ahn et al. 2015). We have previously reported that, although cysteine proteases are not among the most abundant proteins in adult $P$. kellicotti worms, three of them are among the 25 most immunoreactive proteins in adult worm extracts. Indeed, CP-6 was the most abundantly detected immunoreactive protein based on spectral counts (McNulty et al. 2014). The present study showed that a Western blot for IgG4 antibodies to P. kellicotti CP-6 had high sensitivity and specificity, but the parallel IgG assay had low specificity. A previous study with $P$. westermani CP-6 (Western blot for total $\mathrm{IgG}$ ) reported a sensitivity of $77.5 \%$ and a specificity of $96.7 \%$ (Ahn et al. 2015).

Previous studies showed that Western blot assays with crude $P$. westermani or $P$. kellicotti adult worm extracts can be useful for serodiagnosis of paragonimiasis (Fischer et al. 2013; Slemenda et al. 1988). The present study shows that $P$. kellicotti $\mathrm{rCP}-6$ and $\mathrm{rMYO}-1$ can be used in place of native parasite antigen extracts. This may help to standardize the serological diagnosis of paragonimiasis and make testing more widely available. Recombinant CYP-6 and MYO-1 are very promising as serodiagnostic antigens that appear to have excellent sensitivity and specificity when IgG4 (CP-6) or total IgG (MYO-1) antibodies are detected. However, this pilot evaluation only included a limited panel of samples, and larger numbers of samples from different endemic areas will need to be tested to fully evaluate the diagnostic potential of these antigens.

Supplementary Information The online version contains supplementary material available at https://doi.org/10.1007/s00436-020-06990-z.

Acknowledgments The authors would like to thank Drs. Scott Folk (St. Joseph, Missouri), Patricia Wilkins (CDC), and physicians at Washington University School of Medicine for providing sera of individuals with and without proven Paragonimus infection.

Funding The study was supported by a grant from the Barnes-Jewish Hospital Foundation. The Paragonimus genomes project is supported by the National Institute of Health.

\section{Compliance with ethical standards}

Ethical approval Parasite material was produced according to international, national and institutional guidelines. The use of de-identified patients sera for the development of serodiagnostics for helminth parasites was in agreement with the Institutional Review Board of the Washington University School of Medicine in St. Louis.

Conflict of interest The authors declare that they have no conflict of interest.

Open Access This article is licensed under a Creative Commons Attribution 4.0 International License, which permits use, sharing, adaptation, distribution and reproduction in any medium or format, as long as you give appropriate credit to the original author(s) and the source, provide a link to the Creative Commons licence, and indicate if changes were made. The images or other third party material in this article are included in the article's Creative Commons licence, unless indicated otherwise in a credit line to the material. If material is not included in the 
article's Creative Commons licence and your intended use is not permitted by statutory regulation or exceeds the permitted use, you will need to obtain permission directly from the copyright holder. To view a copy of this licence, visit http://creativecommons.org/licenses/by/4.0/.

\section{References}

Ahn CS, Na BK, Chung DL, Kim JG, Kim JT, Kong Y (2015) Expression characteristics and specific antibody reactivity of diverse cathepsin F members of Paragonimus westermani. Parasitol Int 64: 37-42. https://doi.org/10.1016/j.parint.2014.09.012

Bahr NC, Trotman RL, Samman H, Jung RS, Rosterman LR, Weil GJ, Hinthorn DR (2017) Eosinophilic meningitis due to infection with Paragonimus kellicotti. Clin Infect Dis 64:1271-1274. https://doi. org/10.1093/cid/cix102

Blair D (2014) Paragonimiasis. Adv Exp Med Biol 766:115-152. https:// doi.org/10.1007/978-1-4939-0915-5 5

Blair D, Nawa Y, Mitreva M, Doanh PN (2016) Gene diversity and genetic variation in lung flukes (genus Paragonimus). Trans R Soc Trop Med Hyg 110:6-12. https://doi.org/10.1093/trstmh/trv101

Choi JH, Lee JH, Yu HS, Jeong HJ, Kim J, Hong YC, Kong HH, Chung DI (2006) Molecular and biochemical characterization of hemoglobinase, a cysteine proteinase, in Paragonimus westermani. Korean J Parasitol 44:187-196

de Guzman JV, Yu HS, Jeong HJ, Hong YC, Kim J, Kong HH, Chung DI (2007) Molecular characterization of two myoglobins of Paragonimus westermani. J Parasitol 93:97-103. https://doi.org/10. 1645/GE-846R3.1

Feng Y, Furst T, Liu L, Yang GJ (2018) Estimation of disability weight for paragonimiasis: a systematic analysis. Infect Dis Poverty 7:110. https://doi.org/10.1186/s40249-018-0485-5

Fischer PU, Weil GJ (2015) North American paragonimiasis: epidemiology and diagnostic strategies. Expert Rev Anti-Infect Ther 13:779786. https://doi.org/10.1586/14787210.2015.1031745

Fischer K, Beatty WL, Jiang D, Weil GJ, Fischer PU (2011a) Tissue and stage-specific distribution of Wolbachia in Brugia malayi. PLoS Negl Trop Dis 5:e1174. https://doi.org/10.1371/journal.pntd. 0001174

Fischer PU, Curtis KC, Marcos LA, Weil GJ (2011b) Molecular characterization of the North American lung fluke Paragonimus kellicotti in Missouri and its development in Mongolian gerbils. Am J Trop Med Hyg 84:1005-1011. https://doi.org/10.4269/ajtmh.2011.110027

Fischer PU, Curtis KC, Folk SM, Wilkins PP, Marcos LA, Weil GJ (2013) Serological diagnosis of North American Paragonimiasis by Western blot using Paragonimus kellicotti adult worm antigen. Am J Trop Med Hyg 88:1035-1040. https://doi.org/10.4269/ajtmh. $12-0720$

Fischer K, Tkach VV, Curtis KC, Fischer PU (2017) Ultrastructure and localization of Neorickettsia in adult digenean trematodes provides novel insights into helminth-endobacteria interaction. Parasit Vectors 10:177. https://doi.org/10.1186/s13071-017-2123-7

Furst T, Keiser J, Utzinger J (2012) Global burden of human food-borne trematodiasis: a systematic review and meta-analysis. Lancet Infect Dis 12:210-221. https://doi.org/10.1016/S1473-3099(11)70294-8

GBD 2017 Disease and Injury Incidence and Prevalence Collaborators (2018) Global, regional, and national incidence, prevalence, and years lived with disability for 354 diseases and injuries for 195 countries and territories, 1990-2017: a systematic analysis for the
Global Burden of Disease Study 2017. Lancet 392:1789-1858. https://doi.org/10.1016/S0140-6736(18)32279-7

Ikeda T (1998) Cystatin capture enzyme-linked immunosorbent assay for immunodiagnosis of human paragonimiasis and fascioliasis. Am J Trop Med Hyg 59:286-290

Ikeda T, Oikawa Y, Nishiyama T (1996) Enzyme-linked immunosorbent assay using cysteine proteinase antigens for immunodiagnosis of human paragonimiasis. Am J Trop Med Hyg 55:435-437

Keiser J, Utzinger J (2009) Food-borne trematodiases. Clin Microbiol Rev 22:466-483. https://doi.org/10.1128/CMR.00012-09

Kim TS, Na BK, Park PH, Song KJ, Hontzeas N, Song CY (2000) Cloning and expression of a cysteine proteinase gene from Paragonimus westermani adult worms. J Parasitol 86:333-339. https://doi.org/10.1645/0022-3395(2000)086[0333:CAEOAC]2.0. $\mathrm{CO} ; 2$

Kim TY, Joo IJ, Kang SY, Cho SY, Hong SJ (2002a) Paragonimus westermani: molecular cloning, expression, and characterization of a recombinant yolk ferritin. Exp Parasitol 102:194-200

Kim TY, Joo IJ, Kang SY, Cho SY, Kong Y, Gan XX, Sukomtason K, Sukomtason K, Hong SJ (2002b) Recombinant Paragonimus westermani yolk ferritin is a useful serodiagnostic antigen. J Infect Dis 185:1373-1375. https://doi.org/10.1086/339880

Lane MA, Marcos LA, Onen NF, Demertzis LM, Hayes EV, Davila SZ, Nurutdinova DR, Bailey TC, Weil GJ (2012) Paragonimus kellicotti flukes in Missouri, USA. Emerg Infect Dis 18:1263-1267. https:// doi.org/10.3201/eid1808.120335

Martin J et al (2015) Helminth.net: expansions to Nematode.net and an introduction to Trematode.net. Nucleic Acids Res 43:D698-706. https://doi.org/10.1093/nar/gku1128

Martin J, Tyagi R, Rosa BA, Mitreva M (2018) A multi-omics database for parasitic nematodes and trematodes methods. Mol Biol 1757: 371-397. https://doi.org/10.1007/978-1-4939-7737-6 13

McNulty SN, Fischer PU, Townsend RR, Curtis KC, Weil GJ, Mitreva M (2014) Systems biology studies of adult paragonimus lung flukes facilitate the identification of immunodominant parasite antigens. PLoS Negl Trop Dis 8:e3242. https://doi.org/10.1371/journal.pntd. 0003242

McNulty SN et al (2017) Genomes of Fasciola hepatica from the Americas reveal colonization with Neorickettsia endobacteria related to the agents of potomac horse and human sennetsu fevers. PLoS Genet 13:e1006537. https://doi.org/10.1371/journal.pgen.1006537

Na BK, Kim SH, Lee EG, Kim TS, Bae YA, Kang I, Yu JR, Sohn WM, Cho SY, Kong Y (2006) Critical roles for excretory-secretory cysteine proteases during tissue invasion of Paragonimus westermani newly excysted metacercariae. Cell Microbiol 8:1034-1046. https:// doi.org/10.1111/j.1462-5822.2006.00685.x

Park H, Hong KM, Sakanari JA, Choi JH, Park SK, Kim KY, Hwang HA, Paik MK, Yun KJ, Shin CH, Lee JB, Ryu JS, Min DY (2001) Paragonimus westermani: cloning of a cathepsin F-like cysteine proteinase from the adult worm. Exp Parasitol 98:223-227. https:// doi.org/10.1006/expr.2001.4634

Rosa BA, Choi YJ, McNulty SN, Jung H, Martin J, Agatsuma T, Sugiyama H, le TH, Doanh PN, Maleewong W, Blair D, Brindley PJ, Fischer PU, Mitreva M (2020) Comparative genomics and transcriptomics of 4 Paragonimus species provide insights into lung fluke parasitism and pathogenesis. Gigascience 9. https://doi.org/ 10.1093/gigascience/giaa073

Slemenda SB, Maddison SE, Jong EC, Moore DD (1988) Diagnosis of paragonimiasis by immunoblot. Am J Trop Med Hyg 39:469-471

Wang X, Chen W, Huang Y, Sun J, Men J, Liu H, Luo F, Guo L, Lv X, Deng C, Zhou C, Fan Y, Li X, Huang L, Hu Y, Liang C, Hu X, Xu J, Yu X (2011) The draft genome of the carcinogenic human liver 
fluke Clonorchis sinensis. Genome Biol 12:R107. https://doi.org/10. 1186/gb-2011-12-10-r107

Yang SH et al (2004) Cloning and characterization of a new cysteine proteinase secreted by Paragonimus westermani adult worms. Am J Trop Med Hyg 71:87-92

Yoonuan T, Nuamtanong S, Dekumyoy P, Phuphisut O, Adisakwattana $P$ (2016) Molecular and immunological characterization of cathepsin L-like cysteine protease of Paragonimus pseudoheterotremus. Parasitol Res 115:4457-4470. https://doi.org/10.1007/s00436-0165232-x

Young ND, Nagarajan N, Lin SJ, Korhonen PK, Jex AR, Hall RS, SafaviHemami H, Kaewkong W, Bertrand D, Gao S, Seet Q, Wongkham S, Teh BT, Wongkham C, Intapan PM, Maleewong W, Yang X, Hu
M, Wang Z, Hofmann A, Sternberg PW, Tan P, Wang J, Gasser RB (2014) The Opisthorchis viverrini genome provides insights into life in the bile duct. Nat Commun 5:4378. https://doi.org/10.1038/ ncomms 5378

Yu S, Zhang X, Chen W, Zheng H, Ai G, Ye N, Wang Y (2017) Development of an immunodiagnosis method using recombinant PsCP for detection of Paragonimus skrjabini infection in human. Parasitol Res 116:377-385. https://doi.org/10.1007/s00436-016$5300-2$

Publisher's note Springer Nature remains neutral with regard to jurisdictional claims in published maps and institutional affiliations. 